\title{
Towards a cognitive stimulation program using an errorless learning paradigm in amnestic mild cognitive impairment
}

\author{
Léonie Jean' \\ Martine Simard' \\ Robert van Reekum² \\ Marie-Ève Bergeron' \\ 'School of Psychology, Laval \\ University, Quebec City, Quebec, \\ Canada; ${ }^{2}$ Department of Psychiatry \\ and Kunin-Lunenfeld Applied Research \\ Unit, Baycrest Centre for Geriatric \\ Care, Toronto, Canada
}

\begin{abstract}
Considering the high risk for amnestic mild cognitive impairment (A-MCI) individuals to progress towards dementia, it is crucial to study the efficacy of innovative treatment strategies such as cognitive stimulation techniques. The present study is a case report of two individuals presenting with A-MCI who were enrolled in a memory training program. After a broad neuropsychological assessment, the two participants were trained with an errorless (EL) learning paradigm on an individual basis, twice a week, over three weeks. Two follow-up sessions took place one and five weeks after the end of the training. Results showed that the program was well tolerated and feasible, and enhanced daily memory abilities. For the second participant only, a re-evaluation of her cognitive profile was completed 23 months after her first assessment and training. In addition, EL was directly compared with a control condition using an errorful (EF) learning paradigm to teach her new names over two sessions (one session for each condition). Her improvement on the trained material supported the preliminary efficacy of EL compared with EF for learning episodic material. These results are compatible with previous work that has preliminarily demonstrated the efficacy of an EL paradigm in patients with dementia.
\end{abstract}

Keywords: errorless learning, training, memory, elderly, MCI

\section{Introduction}

The prevalence of Alzheimer's disease (AD) and other dementias continues to increase in Western countries, including Canada (Canadian Study of Health and Aging 1994). The study of the prodromal phases of dementia thus becomes mandatory. In this regard, mild cognitive impairment (MCI) is currently conceptualized as an intermediate state between normal aging and early dementia (Petersen et al 1999; Arnaiz and Almkvist 2003; Petersen 2004) in some patients. Each year, approximately $8 \%$ to $20 \%$ of MCI individuals receive a diagnosis of AD or other dementias (Petersen et al 1999; Larrieu et al 2002; Lehrner et al 2005; Ishikawa et al 2006) depending on which MCI criteria are applied.

The most commonly used MCI criteria, for research purposes, typically include a complaint about memory loss, objective memory impairment (ie, at least 1.5 standard deviation [SD] below the mean of a group matched for age and education on a test of episodic memory), and otherwise relatively preserved cognitive and functional abilities, in the absence of dementia (Petersen et al 1999). More recently, the Petersen's "Flow chart of decision process" (Petersen 2004) distinguishes four MCI subtypes that may be useful for diagnostic and prognostic purposes: 1) Amnestic MCI (A-MCI) single domain (impairment of memory only); 2) Amnestic MCI multiple domain (dominant impairment of memory along with an alteration in one or more other cognitive domains); 3) Nonamnestic MCI single domain (impairment of a single nonmemory
Correspondence: Martine Simard School of Psychology, Laval University, Quebec City, QC, CANADA, GIK 7P4 Tel + I 4186562131 ext. I2193 Fax +| 4186563646

Email martine.simard@psy.ulaval.ca 
domain); and 4) Nonamnestic MCI multiple domain (impairment of two or more nonmemory domains). The two A-MCI subtypes are considered to be etiologically linked with AD or vascular dementia (VaD) (Petersen et al 2001; Rasquin et al 2005; Zanetti et al 2006).

Although the application of A-MCI criteria remains a clinical challenge, they are reliable for research purposes (Grundman et al 2004), and present good sensitivity and specificity namely when non memory cognitive domains are considered for diagnosis purposes (Artero et al 2006). Thus the two cases reported in the present paper were diagnosed using the Petersen A-MCI criteria.

Previous studies using cognitive training techniques in mild to moderate AD have shown preliminary tolerability, feasibility, and efficacy (Grandmaison and Simard 2003; Bier et al 2005). It is thus of interest to evaluate the most promising of these techniques in individuals with A-MCI. The errorless learning (EL) (Wilson et al 1994) and spaced retrieval (SR) (Camp 1989; Camp and McKitrick 1992) techniques have been reported to be the best training methods to enhance memory in mild to moderate dementia according to preliminary data (De Vreese et al 2001; Grandmaison and Simard 2003; Bier et al 2005). Briefly, the EL technique aims at keeping errors to a minimum during learning (Baddeley and Wilson 1994), whereas the SR technique aims at gradually increasing the delay between each recall (Camp et al 1996). These two strategies can be used together because it is suggested that the first technique (EL) supports the encoding phase in the way that the information is correctly learned (by reducing or eliminating errors), while the second technique (SR) supports the recall process by repeating the correct information (if an error is made, it is immediately corrected) at increasingly longer intervals (Bier et al 2002).

In the past few years, EL learning was shown to be superior for learning face-name associations in normal aging (Kessels and de Haan 2003), and in dementia (Ruis and Kessels 2005). The efficacy of training programs using an EL paradigm was preliminarily demonstrated in studies with AD patients. In a series of single and multiple case studies (Clare et al 1999, 2000, 2001, 2002, 2003), Clare and her collaborators showed the superiority of this method over EF for learning everyday memory material in mild to moderate AD patients. Some cognitive intervention programs have been developed and evaluated in A-MCI participants (Rapp et al 2002; Belleville et al 2006). In these two studies, the training was multi-faceted, and targeted attention as well as memory in general. The intervention period lasted between 6 (Rapp et al 2002) and 8 weeks (Belleville et al 2006) with a 2-hour group session taking place each week. During these sessions, several stimulation, relaxation and cognitive training techniques, such as computer-assisted attentional training, visual imagery, method of loci, relaxation skill, categorization, etc., were administered. The authors of these two studies do not mention if they applied EL or EF learning paradigms. Belleville and colleagues (2006) showed that MCI individuals, who received the intervention, improved on tasks of episodic and subjective memory. Rapp and colleagues (2002) found a difference in memory appraisal; the treated group showed better perception regarding their memory functioning than did controls. The cognitive intervention programs utilized in these two studies were multi-faceted, and did not specifically target memory systems. It is therefore impossible to determine which component(s) of the programs had an impact on memory. In addition, it may be that greater efficacy would be seen if the intervention specifically targeted memory systems. Another possible constraint on the efficacy of these programs was that they were both offered as group sessions (the two programs were not individualized to each individual's difficulties). One could argue that greater efficacy may perhaps be expected in treatment programs tailored to the individual's impairments, especially in the context of a potentially progressive condition which will eventually lead to more complex difficulties in everyday functioning, as observed in AD. Since individuals are different, and thus have different interests and activities in life, one may assume that day-to-day troubles, even though they are caused at least in part by memory problems, can be of different nature (eg, difficulty recalling names, difficulty with oven functioning, difficulty with medication management, etc). One can therefore suggest that individually tailored programs could be of particular interest with A-MCI individuals who are at risk to progress towards dementia.

Only one study applying an EL paradigm to the A-MCI population has been published to date (Akhtar et al 2006). Akhtar and collaborators (2006) enrolled 16 patients with A-MCI (single domain), and 16 healthy older adults, and trained them over a single experimental individual session lasting up to 60 minutes. Results showed the superiority of EL over EF in A-MCI participants in performing a wordcompletion task. The authors outlined the fact that participants were aware of the benefits of EL by expecting a higher capacity to recall words learned under this condition (Akhtar et al 2006). This research, however, was not a clinical intervention study as it only involved one experimental session, and did not include a follow-up session to investigate the potential preservation of the training benefits. In addition, 
the content of the training (ie, the word list), was not selected according to the participants' memory complaints; it was instead a laboratory task with little ecological validity. It is somewhat uncommon in everyday life to learn a list of words with little or no association between each other, as opposed, for example, to a grocery list or a list of items to prepare for a trip. One can thus argue that the task used by Akhtar and colleagues (2006) was not an appropriate target for an everyday generalization of training. On the contrary, difficulties recalling people's names are frequent complaints and objective deficits described in elderly individuals (Maylor and Valentine 1992; Evrard 2002; Rendell et al 2005).

Despite these limitations, EL in the A-MCI population represents a promising research avenue. The application of this technique seems suitable in the two A-MCI subtypes defined by Petersen (2004), considering that the first cognitive impairment in AD is usually a severe deficit of verbal episodic memory (Petersen et al 1994; Desgranges et al 1996; Small et al 1997; Nestor et al 2006). In view of the high risk to progress towards $\mathrm{AD}$ or $\mathrm{VaD}$ in A-MCI individuals (Petersen 2004; Rasquin et al 2005), it is therefore essential to study the feasibility, tolerability and efficacy of the most promising cognitive training techniques in this particular population. Ultimately, this type of research could lead to the development and/or the enrichment of cognitive training programs specifically designed for dementia at-risk individuals who are aware of their difficulties, and who need help to enhance or maintain their residual memory capacities. Furthermore, since maintaining a certain degree of mental activity was reported to reduce dementia incidence (Wilson et al 2002, Verghese et al 2003; Valenzuela and Sachdev 2006), A-MCI individuals appear to be a particularly valid and important population for applying this kind of training.

\section{Ethical considerations}

This case report study was approved by the Research Ethics Committees of Laval University, and Centre Hospitalier Universitaire de Québec (CHUQ)/ Centre Hospitalier de l'Université Laval (CHUL). Before entering into the study, the participants were fully informed about the research project and the risks of participating in it. They signed an informed consent form that was approved by the Ethics Committees. All nominative data were kept strictly confidential by coding of all documents.

\section{Case A}

Participant A was a 59 year-old male at the time he was referred to our research team by a Memory Disorders Clinic, following his neurological and psychiatric examination. He had 6 years of formal education, and he had been retired from work (he worked as a truck driver) for a year. He was living with his wife and had no children. His medical file indicated that he had been treated for 2 years for hypercholesterolemia, but had no changes in his current medication over the last several months. He had no personal history of neurological or psychiatric illness. However, he had a familial history of $\mathrm{AD}$, as both his parents were diagnosed with $\mathrm{AD}$.

A neuropsychological assessment was first conducted at the participants' home over two 2-hour individual sessions, and included the following tests: the Dementia Rating Scale-2 (DRS-2, Jurica et al 2001), Digit Span (Wechsler 1997), California Verbal Learning Test (CVLT, Delis et al 1987), Boston Naming Test (BNT, Kaplan et al 1983), Tower of London (ToL, Culbertson and Zillmer 2000; Deweer et al unpublished data), Trail Making Test (TMT, Delis et al 2001), and Clock Drawing Test (CDT, Freedman et al 1994) (see Table 1). A 10-minute pause was given after the first testing hour and whenever required by the participant. The tests were administered and corrected in a standardized manner according to the instruction manual of each task used.

As shown in Table 1 (see the Participant A column), he performed 1.5 SD below the mean at screening on episodic memory tasks with virtually all CVLT variables scoring in the impaired range. Some aspects of his executive functioning were impaired as well (eg, Number-letter switching vs Motor speed on the TMT). Nevertheless, his global cognitive functioning was well preserved since his MMSE and DRS-2 total scores were within normal range according to his age and level of education. Finally, the scores obtained on the Neuropsychiatric Inventory (NPI, Cummings et al 1994) and the Disability Assessment for Dementia (DAD, Gauthier et al 1997; Gelinas et al 1999) indicated an absence of, respectively, neuropsychiatric symptoms and problems with activities of daily living (ADL). This individual was considered to meet the A-MCI multiple domain subtype criteria.

He was then invited to take part in a 3-week cognitive training program using the EL paradigm twice a week. The main goal of the 6 training sessions was to re-learn face-name associations of 5 famous individuals from the artistic, political or sports fields in the province of Quebec (Canada). The choice of the material to be re-learned was made on the basis of the participant's and his spouse's complaints. The material used for the memory training sessions consists of 5 black and white pictures of famous individuals from the province of Quebec (Canada). The pictures were manipulated with a computer program in order to obtain the same format for 
Table I Screening (diagnostic) neuropsychological test scores

\begin{tabular}{|c|c|c|c|c|c|c|}
\hline \multirow[t]{2}{*}{ Cognitive domains/Tests } & \multicolumn{2}{|c|}{ Participant A } & \multicolumn{2}{|c|}{ Participant B-I } & \multicolumn{2}{|c|}{ Participant B-2 } \\
\hline & $\begin{array}{l}\text { Raw } \\
\text { scores }\end{array}$ & $\begin{array}{l}\mathbf{Z} \\
\text { scores }\end{array}$ & $\begin{array}{l}\text { Raw } \\
\text { scores }\end{array}$ & $\begin{array}{l}\mathbf{Z} \\
\text { scores }\end{array}$ & $\begin{array}{l}\text { Raw } \\
\text { scores }\end{array}$ & $\begin{array}{l}\mathbf{Z} \\
\text { scores }\end{array}$ \\
\hline \multicolumn{7}{|l|}{ Global cognitive functioning } \\
\hline \multicolumn{7}{|l|}{ DRS-2 } \\
\hline Total score (max. I44) & 137 & 0.33 & 142 & 1.0 & 136 & -0.33 \\
\hline Attention & 37 & 1.00 & 35 & 0 & 34 & -0.67 \\
\hline Initiation/Perseveration & 35 & -0.67 & 37 & 0.33 & 32 & -1.33 \\
\hline Construction & 6 & 0 & 6 & 0 & 6 & 0 \\
\hline Conceptualization & 37 & 0 & 39 & 0.67 & 39 & 0.67 \\
\hline Memory & 22 & -1.00 & 25 & 1.0 & 25 & 1.0 \\
\hline \multicolumn{7}{|l|}{ Attention/Working memory } \\
\hline \multicolumn{7}{|l|}{ Digit span } \\
\hline Total score & 13 & -0.67 & 8 & -1.67 & 11 & -1.0 \\
\hline \multicolumn{7}{|l|}{ Episodic memory } \\
\hline \multicolumn{7}{|l|}{ CVLT (French version) } \\
\hline List A - Sum of trials I to 5 & 28 & -1.80 & 40 & -1.37 & 43 & -0.96 \\
\hline List B - Immediate recall & 3 & -1.32 & 5 & -0.64 & 4 & -1.13 \\
\hline List A - Short term free recall & 3 & -1.98 & 6 & -1.62 & 8 & -0.79 \\
\hline List A - Short term cued recall & 5 & -1.92 & 7 & -1.18 & 9 & -1.14 \\
\hline List A - Delayed free recall & 3 & -2.47 & 8 & $-|.3|$ & 7 & -1.66 \\
\hline List A - Delayed cued recall & 5 & -2.30 & 6 & -2.83 & 8 & -1.87 \\
\hline Recognition - True positives & 11 & $P=5$ & 15 & $P=50$ & 14 & $P=25$ \\
\hline Recognition - False positives & 6 & $P=I$ & 0 & $\mathrm{P}=50$ & 1 & $\mathrm{P}=25$ \\
\hline \multicolumn{7}{|l|}{ Semantic memory/Language } \\
\hline BNT (30-item version) & 23 & -1.00 & 28 & 0 & 22 & -2.0 \\
\hline \multicolumn{7}{|l|}{ Verbal fluency } \\
\hline Phonemic & 28 & -0.99 & 14 & -2.20 & 12 & -2.37 \\
\hline Semantic & 17 & -0.47 & 12 & -1.43 & 10 & -1.93 \\
\hline \multicolumn{7}{|l|}{ Executive functions } \\
\hline \multicolumn{7}{|l|}{ Tower of London $\mathrm{Dx}$} \\
\hline Total move score & 28 & 0.13 & 18 & 1.33 & 17 & 1.47 \\
\hline \multicolumn{7}{|l|}{ Trail Making Test } \\
\hline Visual scanning & 28 & -0.67 & 30 & -0.33 & 30 & -0.33 \\
\hline Number sequencing & 63 & -1.67 & 61 & -1.0 & 86 & -2.33 \\
\hline Letter sequencing & 89 & -3.0 & 64 & -0.67 & 70 & -1.0 \\
\hline Number-Letter Switching & 190 & -2.33 & 165 & -1.33 & 213 & -3.0 \\
\hline Motor speed & 33 & 0 & 32 & 0.33 & 29 & 0.67 \\
\hline Switching vs motor speed & -7 & -2.33 & -5 & -1.67 & -11 & -3.0 \\
\hline \multicolumn{7}{|l|}{ Clock Drawing Test } \\
\hline Free drawing & 6 & -1.7 & 10 & 1.14 & 10 & 1.14 \\
\hline
\end{tabular}

Abbreviations: BNT, Boston Naming Test; CVLT, California Verbal Learning Test; DRS-2, Dementia Rating Scale 2nd edition; MMSE, Mini-Mental State Examination; P, Percentile.

each picture $(6.5 \mathrm{~cm} \times 9.5 \mathrm{~cm})$. This format is the same used by Wilson and colleagues (1985) in the "Faces" subtest of the Rivermead Behavioral Memory Test (RBMT). Each picture was then printed in black and white ink, and pasted on card board $(7.5 \mathrm{~cm} \times 10.5 \mathrm{~cm})$. The face-name associations used for the cognitive training differed between Participant A and Participant $\mathrm{B}$ because the selection of the associations was based on the individual's difficulties recalling famous personalities' names. Thus, at the first training session, 15 pictures covering 3 domains (Arts and Culture, Politics, and Sports) were presented in the order chosen by the participant given his or her interest regarding these 3 domains (from the domain of highest interest to the domain of lowest interest). The participant was then asked to give the first name, the family name and to briefly describe the occupation (eg, singer, politician, etc.) of the person (face) who appeared on the picture. The order of the five target pictures was chosen as follows for subsequent training, based on the performance of the participant at baseline across the three domains taken together: the first two pictures for which the participant was not able to tell the first name or the family name of the individual showed on the picture (ie, for one picture, he had to 
tell the first name or the family name); the first two pictures for which the participant was not able to tell the first name and the family name but demonstrated that he/she knew the occupation of the personality; the first picture for which the participant said that he/she did not know the name nor the occupation of the personality face showed. The order mentioned previously was only to select the pictures on which the participant was trained. Once they were selected, the same five pictures were always used for the subsequent trials, but they were presented in a different order at each trial to avoid an order learning effect (eg, to avoid participants associating the first picture presented with the same name).

The EL paradigm included 5 learning trials and 4 different delays $(2,4,8$, and 16 minutes). The training sessions took place on an individual basis, twice a week, during three weeks, at the participant's home. Each of these 6 training sessions lasted 45 minutes. Each target picture involved in the face-name association learning was presented one at a time, and the participant was asked: "Can you tell me the name of this person? If you are not sure of your response, please do not guess, just tell me that you don't know. I will then give you the correct answer." This procedure was applied for the 5 learning trials included in each session. The items were presented in a different order at each trial. Following the 5 EL trials, additional trials were completed in order to apply the SR technique. The instructions for the SR paradigm were the same as those used for the EL trials. However, the time intervals between recall trials were manipulated based on the participant's performance. If an error was made during a recall trial, the time interval in minutes was reduced to the one previously succeeded at, and the following time intervals were gradually increased by half of the next time interval, once again. For example, if the 4-minute recall trial was failed, (ie, the participant didn't correctly recall $100 \%$ of the facename associations), the next recall trial took place following a 2-minute interval. If this trial was successful (100\% items recalled), the next recall trial took place following a 3-minute interval (since the original interval difference was 2 minutes, the increase was half of this interval, or one minute more) and, if successful, the next trial would take place following a 4-minute interval. The session ended when: 1) 6 trials of SR were completed, successfully or not, or 2) when the largest recall interval (16 minutes) was achieved without errors. At the next training session, the SR trials began with the largest time interval succeeded at, in the previous session. The participants' answers were recorded on an experimental measure named the "Training Measure" (TM). The TM was composed of 5 boxes, each box corresponding to each EL trial, and another box corresponding to the SR paradigm. The answers given by the participants were recorded on this form. The score on this measure represents the sum of the correct answers provided without cueing on each trial in every session.

Figure 1 illustrates the scores obtained on the target material at each trial of the 6 training and 2 follow-up sessions. Participant A was able to recall, without errors, 20\% of the face-name associations at the first trial (S1-1). A marked improvement was then observed (20\% to $90 \%)$ within the first session (S1-1 to S1-5). At the beginning of the second session (S2-1), he showed a slight deterioration compared with S1 but increased his performance across trials (S2-1 to S2-5). However, variable performance until the last training session (S6) was observed. At the end of the three-week intervention period $(\mathrm{S} 6-5=100 \%)$, the proportion of material he was able to correctly recall increased by $400 \%$ compared with baseline ( $\mathrm{S} 1-1=20 \%)$. He was thus capable of remembering all the target material at the end of the training program. His performance was maintained at one-week follow-up (FU1; $100 \%$ recalled), whereas a slight decline was observed at five-week follow-up (FU2; 80\% recalled).

Regarding the SR paradigm, Figure 2 shows the longest delays reached at each of the training sessions (S1-S5). The participant presented a variable performance with a noticeable decrease on S4 followed by a marked peak on S5.

Participant A was also assessed at baseline and at the two follow-up sessions using the Mini-Mental State Examination (MMSE, Folstein et al 1975) and the RBMT (Wilson et al 1985). The follow-up evaluations were completed one and five weeks after completion of the training program. Table 2 presents raw and $\mathrm{Z}$ scores obtained respectively on the MMSE and RBMT. Improvement was obtained on neither the MMSE nor the RBMT at FU1. Although raw scores increased on the two RBMT measures between baseline and FU1, standard scores remained in the impaired range. Nevertheless, a marked improvement was seen on the RBMT Standardized Profile Score with a Z score increase of 1.16 at FU2, compared with baseline. A mild amelioration was also seen on the RBMT Screening total score from baseline to FU2 with an increase of 0.32 ( $\mathrm{Z}$ score). At FU2, this participant also improved his MMSE total score by +0.69 (Z score) compared with baseline.

\section{Case B}

\section{First participation}

Participant B was a 66 year-old woman with 12 years of formal education. She directly contacted the research team after she saw a public announcement of the study. She was 


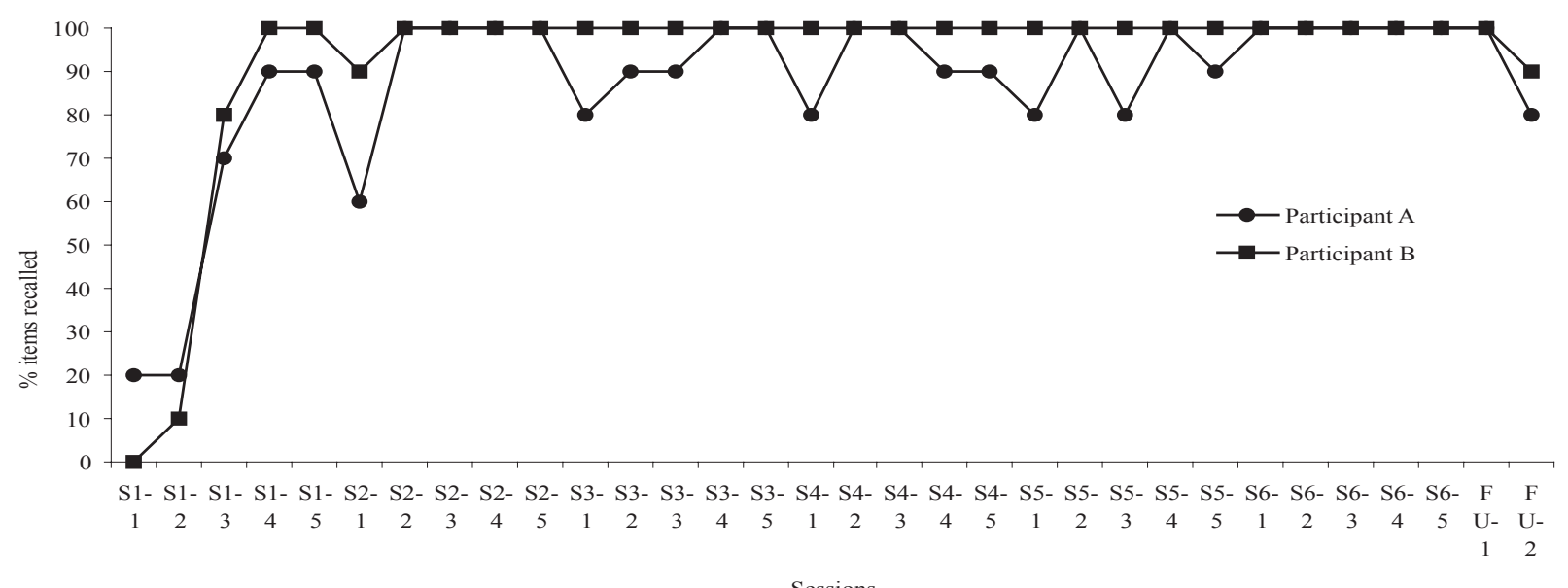

Sessions

Figure I Percentage of items correctly recalled across the training sessions (SI-S6) and at follow-ups (FUI and FU2).

Note: SI-I to S6-5 represents the percentage of items correctly recalled at each trial of every training session in the errorless learning condition.

not married, had no children, and lived alone in an apartment when she was met for the first time by the research team. She had worked all her life as an administrative secretary, but was retired from work for the past two years. She had no personal or familial history of neurological or psychiatric illness and no medical problems at the time of her first participation.

She first underwent the same neuropsychological evaluation as Participant A. As shown in Table 1 (see the Participant B-1 column), she presented with significant alterations in episodic memory, attention, and phonemic fluency. Cognitive flexibility was also impaired. However, her global cognitive functioning was well preserved (per her MMSE and DRS-2 Z scores). Because she lived alone, and had no close relative who could complete the NPI and the DAD, no data are available on these two measures for this participant. Nonetheless, the neuropsychiatric symptoms and ADL abilities were systematically investigated through a clinical interview. She did not show any neuropsychiatric symptoms or ADL problems. She was thus considered to meet the A-MCI multiple domain subtype criteria.

She also took part in a 3-week memory program, the same as designed for participant A and described in details above.

Participant A $\square$ Participant B

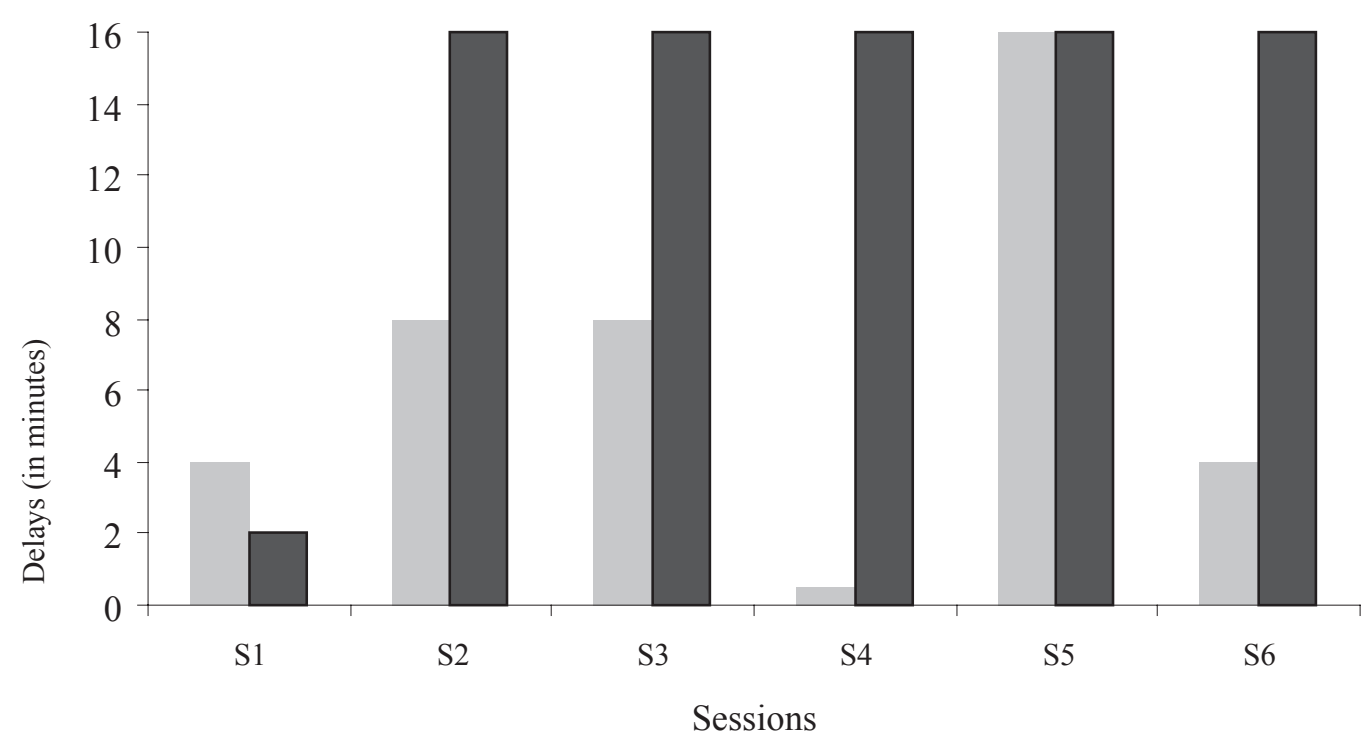

Figure 2 Longest delays reached at each training session (SI-S6). 
Table 2 MMSE and RBMT results at baseline and follow-up (FU) sessions

\begin{tabular}{|c|c|c|c|c|c|c|c|c|}
\hline & \multicolumn{3}{|c|}{ Participant A } & \multicolumn{3}{|c|}{ Participant B-I } & \multicolumn{2}{|c|}{ Participant B-2 } \\
\hline & Baseline & FU I & FU 2 & Baseline & FU I & FU 2 & Baseline & FU I \\
\hline & $\begin{array}{l}\text { Raw score } \\
\text { (Z score) }\end{array}$ & $\begin{array}{l}\text { Raw score } \\
\text { (Z score) }\end{array}$ & $\begin{array}{l}\text { Raw score } \\
\text { (Z score) }\end{array}$ & $\begin{array}{l}\text { Raw score } \\
\text { (Z score) }\end{array}$ & $\begin{array}{l}\text { Raw score } \\
\text { (Z score) }\end{array}$ & $\begin{array}{l}\text { Raw score } \\
\text { (Z score) }\end{array}$ & $\begin{array}{l}\text { Raw score } \\
\text { (Z score) }\end{array}$ & $\begin{array}{l}\text { Raw score } \\
\text { (Z score) }\end{array}$ \\
\hline \multicolumn{9}{|l|}{ MMSE $^{a}$ (Folstein et al 1975) } \\
\hline Total score (max. 30) & $27(0.34)$ & $26(0)$ & $29(1.03)$ & $29(0.7 \mathrm{I})$ & $29(0.7 \mathrm{I})$ & $29(0.7 I)$ & $29(0.7 I)$ & $28(0)$ \\
\hline \multicolumn{9}{|l|}{ 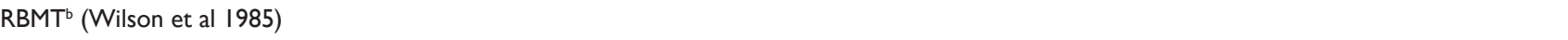 } \\
\hline Standardized profile score (max. 12) & $2(<-3.0)$ & $6(<-3.0)$ & $8(-1.84)$ & $9(-1.13)$ & II (0.28) & $6(\leq 3.0)$ & $10(-0.43)$ & II (0.28) \\
\hline Screening score $(\max .24)$ & $13(<-3.0)$ & $16(<-3.0)$ & $17(-2.68)$ & $19(-1.64)$ & $22(-0.10)$ & $17(-2.68)$ & $22(-0.09)$ & $23(0.42)$ \\
\hline
\end{tabular}

Abbreviations: MMSE, Mini-Mental State Examination; RBMT,Rivermead Behavioural Memory Test.

Notes: ${ }^{\mathrm{T}}$ The MMSE Z scores were calculated based on the normative data of Crum et al (1993); ${ }^{\mathrm{b}}$ The RBMT Z scores were calculated based on the normative data of Wilson et al (1985).

The same instructions were given to participant B. Figure 2 illustrates the scores obtained on the target material at each of the 6 training and 2 follow-up sessions. She did not recall any names $(0 \%)$ at the first trial (S1-1). A marked improvement was observed $(0 \%$ to $100 \%)$ within the first session (S1-1 to S1-5). At the beginning of the second session (S2-1), she showed a slight deterioration but increased her performance across trials (S2-1 to S2-5). Starting with the second trial of the second session (S2-2), she maintained a perfect performance ( $100 \%$ items recalled) until the second follow-up session. At the end of the three-week intervention period ( $\mathrm{S} 6-5=100 \%$ ), the proportion of material correctly recalled increased by $1000 \%$ compared with baseline $(\mathrm{S} 1-1=0 \%)$. She was thus able to recall all the target material at the end of the training program. Her performance was maintained at one-week follow-up (FU1; 100\% recalled), whereas a slight decline was observed at five-week follow-up (FU2; 85\%). Regarding the SR paradigm, Figure 3 shows that she reached the largest interval (16 minutes) on S2 and maintained it until S6.

Table 2 (see the Participant B-1 column) demonstrates no difference between the baseline and follow-up MMSE scores for this participant. An improvement in her performance was however observed between baseline and the first follow-up (FU1) on the RBMT. There was an improvement at FU1 from baseline of $+1.41 \mathrm{Z}$ score on the RBMT Standardized Profile Score, and of $+1.54 \mathrm{Z}$ score on the RBMT Screening total score. However, her scores deteriorated between FU1 and FU2. At FU2, there was a decline in the performance compared with baseline, with a change of $-1.87 \mathrm{Z}$ score on the RBMT standardized profile score, and of $-1.04 \mathrm{Z}$ score on the RBMT screening total score.

\section{Second participation}

In an attempt to characterize her longitudinal cognitive profile, Mrs. B was followed over a 2-year period. At the time of the second screening evaluation (exactly 23 months following the first one), she was 68 years old. Regarding her medical history, the only change reported was that she had been receiving treatment for hypothyroidism over the past year.

She underwent a neuropsychological evaluation with the same tests as those used two years ago. Table 1 (see the Participant B-2 column) shows some episodic memory and verbal fluency impairments, while her attentional ability no longer reached the critical threshold for impairment ( $-1.5 \mathrm{SD}$ below the mean) at the second screening evaluation compared with her previous evaluation 23 months earlier. She lost a few points on the DRS- 2 total score, but her global cognitive functioning was still within normal limits. Phonemic and semantic fluency slightly deteriorated, and continued to be in the impaired range. However, she performed significantly worse on the BNT versus 23 months earlier. She had difficulty with word finding, and phonemic cueing was not always helpful. Regarding everyday memory as assessed by the RBMT (see Table 2), Participant B improved her performance by 4 (+2.57 Z score) and 5 ( $+2.59 \mathrm{Z}$ score) points respectively, on her Standardized profile score and Screening score, between the second follow-up (FU2) of her first participation and the baseline of her second participation 21 months later. Finally, a clinical interview was performed in order to investigate neuropsychiatric symptoms and ADL abilities. No specific problem was detected in these two areas, and compared with the first evaluation, she did not report any change in her daily activities. She was, accordingly, considered to still meet the A-MCI criteria (multiple domain subtype).

She was also invited to undergo two cognitive training sessions, (both taking place on two different days in the same week), using EL learning (first session) and EF learning (second session). The purpose of these sessions was to help her learn ten new names (first and last names) using a control condition, the errorful learning paradigm. Five names were 


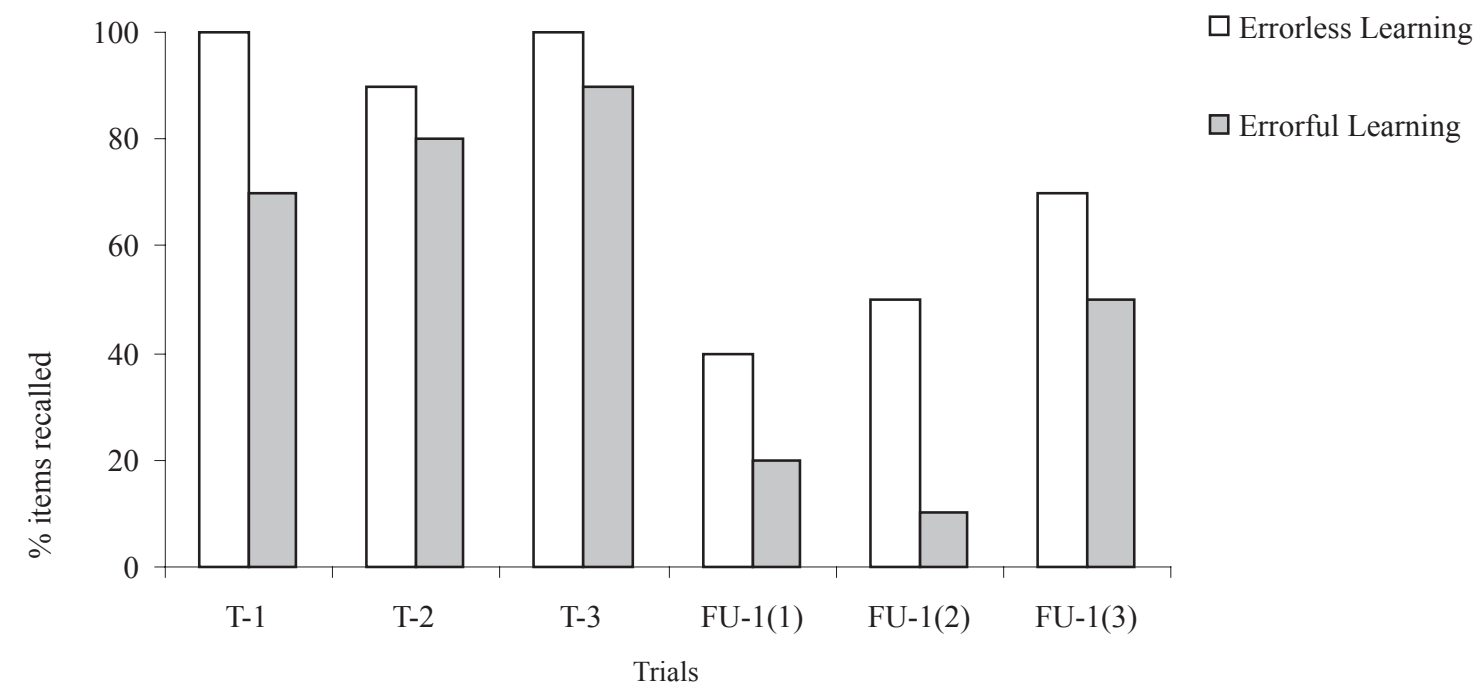

Figure 3 Items correctly recalled in errorless and errorful conditions for Participant B during her second participation. Note: T-I to T-3 = Test trials I to 3; FU-I $(I)=$ First recall trial at follow-up I; FU-I $(2)=$ Second recall trial at follow-up I; FU-I(3)= Third recall trial at follow-up I.

used for the EL session and five names for the EF session. We ensured that it was truly new face-name associations by picking up anonymous pictures on internet and making up fake face-name associations. The material used for training consisted of 10 black and white pictures of individuals unknown to her, and who were not famous people. No occupational information was involved this time. The pictures were manipulated with a computerized program in order to obtain the same format for each picture $(6.5 \mathrm{~cm} \times 9.5 \mathrm{~cm})$. Each picture was then printed in black and white ink and pasted on card board $(7.5 \mathrm{~cm} \times 10.5 \mathrm{~cm})$. For both the EL and EF conditions, first and last names were trained separately, ie, 3 learning trials were completed for each first name followed by 9 test trials. After that, 3 learning trials were performed for each last name, followed by 9 test trials. Subsequently, 3 test trials, combining first and last names, took place.

In the EL condition, the target pictures involved in the face-name association learning were presented one at a time, three times each, and the participant was told: "The first (last) name of this person begins by (the experimenter says the first letter) and his (her) name is ". Please write it down on this piece of paper". The participant was not allowed to see what she wrote during recall after she was asked to write the names down during the learning phase. In the EL trials, the participant was asked: "Can you tell me the first (or last) name of this person? If you are not sure of your response, please do not guess, just tell me that you don't know. I will then give you the correct answer. " In the EF condition, the learning instructions were as following: "The first (last) name of this person begins by (the experimenter says the first letter). Can you guess what is his (her) name?" It was ensured that participant made a minimum of one error. Three guesses were allowed. For example, for each initial (eg L), we had up to three possibilities of names ( $\mathrm{L}=$ Lucy or $\mathrm{L}=$ Lise or $\mathrm{L}=$ Lyne). When we asked the participant to guess, we thus chose a name for the picture among the possibilities already listed on the protocol that wasn't mentioned by the participant on her first guess. This is the same procedure used by Wilson and colleagues (1994). The correct response was then given to the participant: "The first (last) name of this person is Please write it down on this piece of paper. "In the EF trials, the participant was asked: "Can you tell me the first (last) name of this person? If you are not sure, please take a chance." The correct answer was provided if an error was made. As mentioned before, three ultimate trials combining first and last names were performed at the end of each session. For both paradigms, the instructions were the following: "Can you tell me the first and the last names of this person? If you are not sure, please take a chance."

Rates of correct response obtained by Mrs. B on the three test trials at the end of each training session, and at the follow-up evaluation, were calculated. In addition, the rate of forgetting, between training and follow-up sessions, was also computed. Figure 3 illustrates the percentage of items correctly recalled (free recall) under EL and EF conditions. Near perfect scores were obtained with EL learning for the three test trials (T-1, T-2, and T-3) with $100 \%, 90 \%$, and $100 \%$ of the items respectively named (mean $=97 \%$ ).

One follow-up evaluation was then completed, four weeks after the cognitive training sessions, by a research 
assistant who did not evaluate the participant at baseline and did not administer the training sessions. As in Experiment 1, the MMSE and RBMT were administered (see the Participant B-2 column of Table 2). The followup examiner was blinded to the baseline results and did not know which items (names) were trained with which paradigm (EL vs EF). For EL, after a 4-week follow-up, the participant correctly identified $53 \%$ of the names on average, thus indicating a mean forgetting rate of $44 \%$ (mean $=97 \%-53 \%$ ) in long-term memory. For EF learning, $70 \%, 80 \%$ and $90 \%$ of the names were adequately recalled during the three test trials, with a mean of $80 \%$. At follow-up, $27 \%$ of this material was adequately named, with a mean forgetting rate of $53 \%$. She also showed a small drop in MMSE score between baseline (Raw score $=29 / 30$, $\mathrm{Z}$ score $=0.71)$ and follow-up $($ Raw score $=28 / 30, \mathrm{Z}$ Score $=0)$. In contrast, a relative preservation of her performance was found, between baseline and follow-up, on the RBMT. She improved her Screening score from $22(Z$ score $=-0.09)$ to 23 ( $\mathrm{Z}$ score $=+0.42$ ) while her Standardized Profile score showed the same pattern with an improvement of one point from $10(\mathrm{Z}$ score $=-0.43)$ to $11(\mathrm{Z}$ score $=+0.28)$.

\section{Discussion}

To our knowledge, this was the first study to use an EL paradigm, in individuals presenting with A-MCI, to help them learn and recall names. Since difficulties recalling new people's names (episodic content) and famous names (semantic content) are common complaints and an objective deficit not only in A-MCI individuals (Schmand et al 1996; Estevez-Gonzalez et al 2004) but also in elderly people in general (Maylor and Valentine 1992; Evrard 2002; Rendell et al 2005), the training material used in the present case reports may have benefit not only in A-MCI, but in elderly populations as well.

Both participants completed the memory training program without missing any training sessions or any follow-up evaluations. In addition, there was no apparent sign or expression of fatigue at the end of each session, and neither of the participants expressed or showed overwhelming signs of anxiety or distress. They complied with the instructions of the examiner and trainer without difficulty. These observations thus demonstrate the feasibility of the 3-week individual cognitive training program administered twice a week. They were also interviewed at the last follow-up session to investigate their appreciation regarding the cognitive stimulation program. Whereas they knew they had memory difficulties, both participants outlined the fact that the intervention improved their self-confidence concerning their memory functioning by realizing that they could also count on residual memory capacities. These positive comments expressed by the participants at the end of the study highlighted the self-perceived benefits of this intervention.

These case reports addressed the preliminary efficacy of the EL paradigm to help A-MCI individuals re-learn semantic memory information. The proportion of names correctly recalled after the three-week intervention period increased by 400\% (Participant A) and 1000\% (Participant B) compared with baseline. At the first follow-up, the gains of the training were maintained $(100 \%$ of retention registered in both participants). At the second follow-up, Mr. A retained 80\% whereas Mrs. B retained $85 \%$ of the training material. The memory training program thus preliminarily demonstrated some efficacy.

The possible stimulating and nonspecific effects of the memory training on other memory and nonmemory tasks were also assessed. The two participants registered clinically significant improvements in everyday memory functioning, as measured by the RBMT, between the beginning and the end of the training program (FU-1). Practice effects could not entirely explain the improvement observed between baseline and the first follow-up as parallel forms of the RBMT were used. Furthermore, when Wilson and her colleagues (1985) assessed the test re-test reliability of the RBMT in 118 brain damaged patients, they found only a slight, marginal practice effect with an increase of 0.27 raw points for the Screening score and of 0.76 raw points for the Standardized Profile score (RBMT Manual, Wilson et al 1985). Another argument against a practice effect is the significant decline that was registered by Participant $B$ at the second follow-up, 5 weeks following the end of the training. The EL and SR techniques thus appear to have been efficacious in enhancing everyday memory functioning, but the long-term (5-week delay) effects were not clear with an improvement in one case and a decline in the other. Long-term efficacy thus remains questionable and this is a limitation of the present study. In order to enhance or maintain long-term efficacy over weeks and months of this training in the future, additional sporadic sessions could be offered to participants during the year following their participation in the study. This procedure might benefit participants by reminding them of the memory techniques, and prompting them to apply these techniques in their everyday life.

Despite the fact that both participants benefited from the program in re-learning the target material, there was no improvement on their global cognition as assessed by the MMSE between the baseline evaluation and the first follow-up. However, the first participant showed an amelioration of his MMSE score between the two follow-ups. Practice effects 
might perhaps explain this improvement. The lack of improvement on the MMSE scores registered by Participant B during her first participation was expected since the MMSE was not directly trained material. Nonetheless, practice effects could have occurred given the short interval between the evaluations. The absence of practice effects could have been due to a selection bias (normal general cognitive functioning was an inclusion criterion) that possibly created a ceiling effect for Mrs. B baseline MMSE performance. These present case reports thus suggest that the utilization of direct training measures and specific cognitive instruments in the evaluation of the effects of cognitive training in A-MCI is more appropriate than that of tests of general cognitive functioning.

Despite promising findings, the results obtained for Cases A and B (first experiment) are limited by the absence of a betweenor within-subject control condition. For this reason, in the case of Mrs. B, an investigation of the efficacy of EL compared with an errorful condition (within-subject control) was conducted. In addition, because the same individual applied the training techniques and performed the evaluations, experimenter bias could have been introduced. Consequently, follow-up assessments performed by an examiner blind to the results of the baseline evaluation, and to the memory training condition, would enhance the robustness of research and, as such, this approach was utilized for Participant B's second experiment.

Her improvement on the trained items supported the preliminary efficacy of EL, versus EF, for learning episodic material. Our results are thus compatible with previous work that has preliminarily demonstrated the efficacy of an EL paradigm in patients with mild-to-moderate AD (Clare et al 1999; Clare et al 2002; Metzler-Baddeley and Snowden 2005) and A-MCI (Akhtar et al 2006). Moreover, the fact that she maintained, to some extent, the learned material at 4-weeks follow-up is encouraging for this kind of intervention. In this regard, more follow-up sessions would be useful to examine whether gains are maintained over time.

The follow-up of Case B over a two-year period also allowed the collection of longitudinal data regarding her neuropsychological profile. After a 23-month interval, her cognitive profile again met the criteria for A-MCI with relative stability, over time, of the deficits detected at the first evaluation. Some aspects of her cognition, however, worsened, such as confrontation naming capacity measured with the BNT. However, only one participant included in the present study underwent a longitudinal neuropsychological evaluation and this is undoubtedly a major limitation, since some MCI patients improve at follow-up (Larrieu et al 2002).
In conclusion, despite their limitations, the present case reports have shown promising results, especially regarding the difference between EL and EF learning, that support the application and evaluation of this kind of cognitive stimulation program in a large sample using a randomized placebocontrolled design.

\section{Acknowledgments}

Mrs Jean is supported by doctoral training grants awarded by the Alzheimer Society of Canada co-funded by the Fonds de la Recherche en Santé du Québec (FRSQ) and the Institute of Aging, and by the FormSaV Program. Dr Simard is supported by a 2005 National Alliance for Research in Schizophrenia and Depression (NARSAD) Young Investigator Award, and the Centre de Recherche Université Laval Robert Giffard, and is involved in research funded by the CIHR (Institute of Aging). Dr. van Reekum is supported by the Kunin Lunenfeld Applied Research Unit, Baycrest.

Part of these results were presented at the 66th Annual Convention of the Canadian Psychological Association held in Montreal, QC, Canada, June 9-11, 2005, at the International Neuropsychological Society, British Neuropsychological Society and the Division of Neuropsychology of the British Psychological Society Joint Mid-Year Meeting held in Dublin, Ireland, July 6-9, 2005 and at the 18th Annual Meeting of the American Neuropsychiatric Association held in Tucson, Arizona, USA, February 17-20, 2007.

\section{References}

Akhtar S, Moulin CJ, Bowie PC. 2006. Are people with mild cognitive impairment aware of the benefits of errorless learning? Neuropsychological Rehabilitation, 16:329-46.

Arnaiz E, Almkvist O. 2003. Neuropsychological features of mild cognitive impairment and preclinical Alzheimer's disease. Acta Neurol Scand Suppl, 179:34-41.

Artero S, Petersen R, Touchon J, et al. 2006. Revised Criteria for Mild Cognitive Impairment: Validation within a Longitudinal Population Study. Dement Geriatr Cogn Disord, 22:465-70.

Baddeley A, Wilson BA. 1994. When implicit learning fails: amnesia and the problem of error elimination. Neuropsychologia, 32:53-68.

Belleville S, Gilbert B, Fontaine F, et al. 2006. Improvement of episodic memory in persons with mild cognitive impairment and healthy older adults: evidence from a cognitive intervention program. Dement Geriatr Cogn Disord, 22:486-99.

Bier N, Gagnon L, Desrosiers J. 2005. Hétérogénéité des déficits cognitifs dans la démence de type Alzheimer et prise en charge cognitive de la mémoire : particularités et enjeux méthodologiques. Canadian Journal on Aging, 24:275-84.

Bier N, Vanier M, Meulemans T. 2002. Errorless learning: A method to help amnesic patients learn new information. Journal of Cognitive Rehabilitation, 20:12-8.

Camp CJ. 1989. Facilitation of new learning in Alzheimer's disease. Memory, Aging and Dementia. Gilmore GC, J.Whitehorse P and Wykle MLNew York:: Springer Publishing Company:212-25.

Camp CJ, Foss JW, O'Hanlon AM, et al. 1996. Memory interventions for persons with dementia. Applied Cognitive Psychology, 10:193-210. 
Camp CJ, McKitrick LA. 1992. Memory interventions in Alzheimer's-type dementia populations: methodological and theoretical issues. Everyday Memory and Aging: Current Research and Methodologies. West RL and Sinnott JDNew York: Springer-Verlag.:155-72.

Clare L, Wilson BA, Breen K, et al. 1999. Errorless learning of face-name associations in early Alzheimer's disease. Neurocase, 5:37-46.

Clare L, Wilson BA, Carter G, et al. 2000. Intervening with everyday memory problems in dementia of Alzheimer type: an errorless learning approach. J Clin Exp Neuropsychol, 22:132-46.

Clare L, Wilson BA, Carter G, et al. 2003. Cognitive rehabilitation as a component of early intervention in Alzheimer's disease: a single case study. Aging Ment Health, 7:15-21.

Clare L, Wilson BA, Carter G, et al. 2001. Long-term maintenance of treatment gains following a cognitive rehabilitation intervention in early dementia of Alzheimer type: a single case study. Neuropsychological Rehabilitation, 11:477-94.

Clare L, Wilson BA, Carter G, et al. 2002. Relearning face-name associations in early Alzheimer's disease. Neuropsychology, 16:538-47.

Crum RM, Anthony JC, Bassett SS, et al. 1993. Population-based norms for the Mini-Mental State Examination by age and educational level. JAMA, 269:2386-91.

CSHA. 1994. The Canadian Study of Health and Aging: Risk factors for Alzheimer's disease in Canada. Neurology, 44:2073-80.

Culbertson WC, Zillmer EA. 2000. Tower of London. Toronto, ON: MultiHealth Systems.

Cummings JL, Mega M, Gray K, et al. 1994. The Neuropsychiatric Inventory: comprehensive assessment of psychopathology in dementia. Neurology, 44:2308-14.

De Vreese LP, Neri M, Fioravanti M, et al. 2001. Memory rehabilitation in Alzheimer's disease: a review of progress. Int J Geriatr Psychiatry, 16:794-809.

Delis DC, Kaplan E, Kramer JH. 2001. The Delis-Kaplan Executive Function System. San Antonio, TX: The Psychological Corporation.

Delis DC, Kramer JH, Kaplan E, et al. 1987. California Verbal Learning Test: Adult version manual. San Antonio, TX: The psychological Corporation.

Desgranges B, Eustache F, Rioux P, et al. 1996. Memory disorders in Alzheimer's disease and the organization of human memory. Cortex, $32: 387-412$.

Deweer B, Benoit N, Coyette F, et al. Le California Verbal Learning Test: adaptation française et étalonnage. Unpublished data

Estevez-Gonzalez A, Garcia-Sanchez C, Boltes A, et al. 2004. Semantic knowledge of famous people in mild cognitive impairment and progression to Alzheimer's disease. Dement Geriatr Cogn Disord, 17:188-95.

Evrard M. 2002. Ageing and lexical access to common and proper names in picture naming. Brain Lang, 81:174-9.

Folstein MF, Folstein SE, McHugh PR. 1975. "Mini-mental state". A practical method for grading the cognitive state of patients for the clinician. $J$ Psychiatr Res, 12:189-98.

Freedman M, Leach L, Kaplan E, et al. 1994. Clock drawing: A neuropsychological analysis. New York, NY: Oxford University Press.

Gauthier S, Gelinas I, Gauthier L. 1997. Functional disability in Alzheimer's disease. Int Psychogeriatr, 9 Suppl 1:163-5.

Gelinas I, Gauthier L, McIntyre M, et al. 1999. Development of a functional measure for persons with Alzheimer's disease: the disability assessment for dementia. Am J Occup Ther, 53:471-81.

Grandmaison E, Simard M. 2003. A critical review of memory stimulation programs in Alzheimer's disease. J Neuropsychiatry Clin Neurosci, 15:130-44.

Grundman M, Petersen RC, Ferris SH, et al. 2004. Mild cognitive impairment can be distinguished from Alzheimer disease and normal aging for clinical trials. Arch Neurol, 61:59-66.

Ishikawa T, Ikeda M, Matsumoto N, et al. 2006. A longitudinal study regarding conversion from mild memory impairment to dementia in a Japanese community. Int J Geriatr Psychiatry, 21:134-9.

Jurica PJ, Leitten CL, Mattis S. 2001. Dementia Rating Scale-2, Professional Manual. Lutz, FL: Psychological Assessment Resources, Inc.
Kaplan EF, Goodlass H, Weintraub S. 1983. The Boston Naming Test (2nd edition). Philadelphia: Lea and Febiger.

Kessels RP, de Haan EH. 2003. Mnemonic strategies in older people: a comparison of errorless and errorful learning. Age Ageing, 32:529-33.

Larrieu S, Letenneur L, Orgogozo JM, et al. 2002. Incidence and outcome of mild cognitive impairment in a population-based prospective cohort. Neurology, 59:1594-9.

Lehrner J, Gufler R, Guttmann G, et al. 2005. Annual conversion to Alzheimer disease among patients with memory complaints attending an outpatient memory clinic: the influence of amnestic mild cognitive impairment and the predictive value of neuropsychological testing. Wien Klin Wochenschr, 117:629-35.

Maylor EA, Valentine T. 1992. Linear and nonlinear effects of aging on categorizing and naming faces. Psychol Aging, 7:317-23.

Metzler-Baddeley C, Snowden JS. 2005. Brief report: errorless versus errorful learning as a memory rehabilitation approach in Alzheimer's Disease. J Clin Exp Neuropsychol, 27:1070-9.

Morris JC, Price AL. 2001. Pathological correlates of nondemented aging, mild cognitive impairment, and early-stage Alzheimer's disease. Journal of Molecular Neurosciences, 17:101-18.

Nestor PJ, Fryer TD, Hodges JR. 2006. Declarative memory impairments in Alzheimer's disease and semantic dementia. Neuroimage, 30:1010-20.

Petersen RC. 2004. Mild cognitive impairment as a diagnostic entity. J Intern Med, 256:183-94.

Petersen RC, Doody R, Kurz A, et al. 2001. Current concepts in mild cognitive impairment. Arch Neurol, 58:1985-92.

Petersen RC, Smith GE, Ivnik RJ, et al. 1994. Memory function in very early Alzheimer's disease. Neurology, 44:867-72.

Petersen RC, Smith GE, Waring SC, et al. 1999. Mild cognitive impairment: clinical characterization and outcome. Arch Neurol, 56:303-8.

Rapp S, Brenes G, Marsh AP. 2002. Memory enhancement training for older adults with mild cognitive impairment: a preliminary study. Aging Ment Health, 6:5-11.

Rasquin SM, Lodder J, Visser PJ, et al. 2005. Predictive accuracy of MCI subtypes for Alzheimer's disease and vascular dementia in subjects with mild cognitive impairment: a 2-year follow-up study. Dement Geriatr Cogn Disord, 19:113-9.

Rendell PG, Castel AD, Craik FI. 2005. Memory for proper names in old age: a disproportionate impairment? Q J Exp Psychol A, 58:54-71.

Ruis C, Kessels RP. 2005. Effects of errorless and errorful face-name associative learning in moderate to severe dementia. Aging Clin Exp Res, 17:514-7.

Schmand B, Jonker C, Hooijer C, et al. 1996. Subjective memory complaints may announce dementia. Neurology, 46:121-5.

Small BJ, Herlitz A, Fratiglioni L, et al. 1997. Cognitive predictors of incident Alzheimer's disease: a prospective longitudinal study. Neuropsychology, 11:413-20.

Valenzuela MJ, Sachdev P. 2006. Brain reserve and dementia : A systematic review. Psychol Med, 36:441-454.

Verghese J, Lipton R, Katz M, et al. 2003. Leisure activities and the risk of dementia in the elderly. $N$ Engl J Med, 348:2508-2516.

Wechsler D. 1997. Wechsler Adult Intelligence Scale, 3rd Edition. San Antonio, TX: The Psychological Corporation.

Wilson BA, Baddeley A, Evans J, et al. 1994. Errorless learning in the rehabilitation of memory impaired people. Neuropsychological Rehabilitation, 4:307-26.

Wilson BA, Cockburn J, Baddeley A. 1985. The Rivermead Behavioral Memory Test. Bury St-Edmunds, England: Thames Valley Company.

Wilson RS, Mendes de Leon CF, Barnes LL, et al. 2002. Participation in cognitively stimulating activities and risk of incident Alzheimer disease. JAMA, 287:742-748.

Zanetti M, Ballabio C, Abbate C, et al. 2006. Mild cognitive impairment subtypes and vascular dementia in community-dwelling elderly people: a 3-year follow-up study. J Am Geriatr Soc, 54:580-6. 
